Rabaska

Revue d'ethnologie de l'Amérique française

GENEST, BERNARD. Une saison au bord de l'eau. Lac Magog, un site de villégiature dans les Cantons-de-l'Est. Sherbrooke, Éditions GGC, collection " Patrimoine ", 2003, 221 p. ISBN

2-89444-182-7

\title{
Philippe Dubé
}

Volume 2, 2004

URI : https://id.erudit.org/iderudit/201667ar

DOI : https://doi.org/10.7202/201667ar

Aller au sommaire du numéro

Éditeur(s)

Société québécoise d'ethnologie

ISSN

1703-7433 (imprimé)

1916-7350 (numérique)

Découvrir la revue

Citer ce compte rendu

Dubé, P. (2004). Compte rendu de [GENEST, BERNARD. Une saison au bord de l'eau. Lac Magog, un site de villégiature dans les Cantons-de-l'Est. Sherbrooke, Éditions GGC, collection « Patrimoine ", 2003, 221 p. ISBN 2-89444-182-7].

Rabaska, 2, 216-219. https://doi.org/10.7202/201667ar d'utilisation que vous pouvez consulter en ligne.

https://apropos.erudit.org/fr/usagers/politique-dutilisation/ 
Genest, Bernard. Une saison au bord de l'eau. Lac Magog, un site de villégiature dans les Cantons-de-l'Est. Sherbrooke, Éditions GGC, collection «Patrimoine », 2003, 221 p. ISBN 2-89444-182-7.

Faire l'histoire à partir de matériaux épars est le lot de toute construction quand vient le temps de refaire le passé, celui qui échappe inexorablement au présent malgré les efforts de rétention que déploient les sciences dites 
historiques. Que ce soit l'histoire à proprement parler, l'archéologie, l'histoire de l'art ou encore l'ethnologie, toutes ces disciplines participent à l'entreprise de revisiter l'héritage à travers les traces que laissent sur son passage l'aventure humaine. Parmi ces disciplines que convoque le récit historique, l'ethnologie - celle que plusieurs d'entre nous pratiquent - offre avant tout une qualité du regard qui sait se pencher tout particulièrement sur les personnes en cause. C'est pour ainsi dire le point de départ, l'élément déclencheur, le centre d'intérêt premier de l'enquêteur-ethnographe: celui de prendre contact le plus directement possible avec ceux et celles qui ont été engagés dans l'aventure à l'étude. C'est d'ailleurs ce qui fait habituellement le succès de l'angle ethnographique, notamment la prise en compte prioritaire des acteurs dans tout projet, qu'il soit collectif ou encore individuel. Dans le cas qui nous occupe, Bernard Genest, avec Une saison au bord de l'eau, avait en mains plusieurs éléments pour réussir son entreprise, soit une cuvre ethnohistorique prégnante sur la villégiature qui s'est déroulée au petit lac Magog.

L'auteur a d'abord réussi par la richesse documentaire qu'il a su réunir. Nombre de cartes postales, de photographies tirées d'albums de famille, d'articles ou d'annonces prises dans les journaux locaux, de dépliants publicitaires et de correspondances privées viennent enrichir son récit qui se veut structurant dans le but de circonscrire la mise en place de ce lieu de vacances prisé, en ses débuts, par les gens des Cantons-de-l'Est. Il a su aussi, en bon ethnologue, tirer profit des sources orales qu'il a puisées à même les membres de sa propre famille. Car, en effet, Genest ici raconte en quelque sorte les Genest qui viennent prendre leurs vacances au bord du lac Magog. En fait, Bernard a poursuivi l'œuvre que son père Gaston avait commencée, celle non seulement de colliger des documents mais surtout, la plus difficile, celle de construire un récit sur l'histoire du lieu dit. De ce point de vue, l'auteur a fait le choix d'obéir à une logique temporel linéaire où l'on part des débuts amérindiens de la région que la toponymie des lieux exprime éloquemment (Magog, Memphrémagog, Massawippi, Passumpsic, Scaswaninepus, Mégantic, etc.). Pour finalement terminer l'ouvrage avec « Le Lac Magog aujourd'hui » où, avec une note un brin nostalgique, il rend brièvement compte des derniers développements du « lieu mythique, chargé d'histoire ". Le panorama qu'il propose esquisse une vue d'ensemble fort éclairante sur les développements de cette petite colonie de vacances devenue, avec le temps, un lieu incontournable pour la pratique de la villégiature dans la région sherbrookoise. Une histoire qui s'étale sur un peu plus d'un siècle et qui nous fait revivre à travers les péripéties d'une famille élargie la genèse d'un « petit coin de paradis ».

La démarche d'ensemble tente d'intégrer dans cette revue du lieu les activités qui s'y déroulent. Après avoir traité des origines, il fallait en premier 
lieu aborder la question de l'établissement et de l'aménagement des familles en vacances. L'architecture y est donc étudiée de manière sommaire tout en faisant prendre conscience de l'importance de cette toute première nécessité, même s'il s'agit de villégiature. Puis, dans un enchaînement parfaitement naturel, on distingue les statuts du villégiateur en vacances de celui du touriste en séjour à l'hôtel, question de bien camper les concepts à l'étude. Puis les activités habituelles sont déclinées d'une façon ordonnée, avec la chasse, la pêche, les clubs privés, les plaisirs de l'eau et les joies du plein air. Finalement, les aspects de la vie en vacances liées au calendrier sont approfondis dans la suite par l'évocation du déroulement traditionnel du dimanche et des jours fériés. Et ceci, avant d'aborder les événements qui marquaient régulièrement la belle saison. Et c'est sur cette note presque finale que Bernard Genest termine son étude par un constat actuel qu'il fait en conclusion. Ce tour d'horizon nous propose une manière de « tour du propriétaire " où l'auteur parle de ce qu'il connaît à la fois intimement et de ce qu'il a par la suite apprivoisé professionnellement. Au bout du compte, il procède à une longue entreprise d'objectivation d'une mémoire surtout subjective qu'il tente de redresser dans ses travers par un récit structuré. Et c'est précisément là que loge la qualité centrale et première de ce livre qui se trouve, on l'aura compris, à la jonction d'un grand journal de famille et d'une étude fouillée qui fait intervenir plusieurs champs disciplinaires.

Ce qui m'a le plus interpellé dans cet ouvrage qui a su produire une belle page d'histoire inconnue jusqu'à ce jour, c'est la part plus personnelle de l'auteur. Celle où il fait référence à ses souvenirs d'enfance, dans les encarts du livre notamment, où il livre une matière riche de sa propre mémoire et celle de sa famille. Il ne s'agit pas ici pour ma part d'avoir été motivé par un intérêt curieux pour le plus pittoresque mais plutôt saisi par la découverte d'une mine de souvenirs qui rendent la lecture encore plus vraie. C'est là où l'ethnologue s' « ethnologise » en quelque sorte et donne une matière exaltante à se remémorer en sa compagnie. Pour cette unique raison, l'ouvrage mérite l'attention et sa lecture fera la juste démonstration que la discipline gagne parfois à être dépassée par l'indiscipline du commentaire personnel. Là où la mémoire de l'auteur arrive à rehausser par son propre témoignage la valeur de l'étude en cause. Et c'est certainement la plus grande réussite de l'ouvrage que seul un auteur en pleine maturité peut en somme atteindre. Au-delà de mon appréciation très favorable à l'égard de ce dernier-né de l'ethnologue Bernard Genest, $\mathrm{j}$ 'aimerais lui signaler en toute amitié qu'en avant-propos, là où il déplore justement le fait que peu d'ouvrages traitent du phénomène de la villégiature au Québec, il y a pourtant le mien qui en fait état longuement. En effet, j'ai publié aux Presses de l'Université Laval en 1986 Deux cents ans de villégiature dans Charlevoix et non Deux siècles d'architecture dans 
Charlevoix comme il en est fait mention en note 2 et en bibliographie. Et dans cet ouvrage qui reste le condensé d'une thèse de doctorat, je crois en aborder les divers aspects et en situer surtout l'émergence. Mais ceci est certainement une simple coquetterie d'auteur de ma part qui, de toute manière, n'altère en rien mon jugement quant à la valeur du livre de B.G. D'ailleurs, en guise de conclusion, je dirais que la qualité de la langue qu'il utilise dans son texte fera l'envie de plusieurs. Comme l'historien Fernand Braudel le rappelle avec pertinence: «Or, comme dans tous les débats sérieux, les mots sont rois ». Et j'ajouterais que le maniement du mot juste fait de celui qui en joue avec talent le maître des rois.

Philippe Dubé

Université Laval 